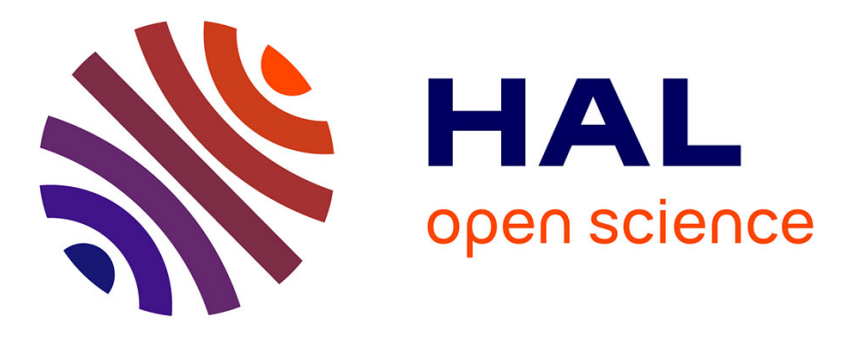

\title{
Improving Turbo Codes for 5G with Parity Puncture-Constrained Interleavers
}

Ronald Garzon Bohorquez, Charbel Abdel Nour, Catherine Douillard

\section{To cite this version:}

Ronald Garzon Bohorquez, Charbel Abdel Nour, Catherine Douillard. Improving Turbo Codes for 5G with Parity Puncture-Constrained Interleavers. ISTC 2016: 9th International Symposium on Turbo Codes \& Iterative Information Processing, Sep 2016, Brest, France. pp.151 - 155, 10.1109/ISTC.2016.7593095 . hal-01421989

\section{HAL Id: hal-01421989 \\ https://hal.science/hal-01421989}

Submitted on 17 Feb 2020

HAL is a multi-disciplinary open access archive for the deposit and dissemination of scientific research documents, whether they are published or not. The documents may come from teaching and research institutions in France or abroad, or from public or private research centers.
L'archive ouverte pluridisciplinaire HAL, est destinée au dépôt et à la diffusion de documents scientifiques de niveau recherche, publiés ou non, émanant des établissements d'enseignement et de recherche français ou étrangers, des laboratoires publics ou privés. 


\title{
Improving Turbo Codes for 5G with Parity Puncture-Constrained Interleavers
}

\author{
Ronald Garzón-Bohórquez, Charbel Abdel Nour, and Catherine Douillard \\ TELECOM Bretagne (Institut MINES-TELECOM), CNRS UMR 6285 Lab-STICC \\ Brest, France \\ Email: \{ronald.garzonbohorquez, charbel.abdelnour, catherine.douillard\}@telecom-bretagne.eu
}

\begin{abstract}
A new method to design punctured Turbo Codes (TCs) with improved performance in both the waterfall and error floor regions is introduced. First, the puncturing pattern is selected by analyzing the constituent code distance spectrum and the TC extrinsic information exchange using uniform interleavers. Then, the interleaver function is defined via a graph-based approach including different design criteria such as minimum span, correlation girth, and puncturing constraints during the design process. An application example is elaborated and compared with the Long Term Evolution (LTE) standard: a significant gain in error rate performance can be observed. An additional benefit of the proposed technique is a significant reduction of the search space for the different interleaver parameters.
\end{abstract}

\section{INTRODUCTION}

In recent years, nomadic users got interested in a larger diversity of applications and services, such as TV or multimedia contents on demand. Accordingly, the future $5^{\text {th }}$ generation of mobile networks (5G) [1] calls for increased data rates and capacity, with an enhanced Quality of Service for different receiver scenarios and applications. To meet such requirements, error-correcting codes able to guarantee very low error rates (down to $10^{-6}$ of Frame Error Rate (FER)) are required. A current communication system such as Long Term Evolution (LTE) [2] cannot guarantee such error rates. First of all, due to the HARQ retransmission mechanism, the targeted error rate in LTE is $10^{-2}$ of FER. In addition, the rate matching mechanism in LTE can cause bad interactions between the code interleaver and the puncturing mechanism, resulting in bad error rate performance at low error rates [3].

The LTE standard adopted a Turbo Code (TC) as channel code [2]. Provided that they would be able to guarantee lower error rates when punctured, TCs could remain promising channel coding candidates for 5G. As introduced by Berrou et al. [4], the minimum Hamming distance $\left(d_{\min }\right)$ of a TC is not only defined by its constituent encoders but also fixed through the TC interleaver. In this study, we investigate the joint design of interleavers and puncturing patterns for TCs in order to guarantee low error floors and good convergence thresholds. As a result, a new puncturing constraint related to parity puncturing is proposed for the design of TC interleavers. This work focuses on the Almost Regular Permutation (ARP) interleaver model [5], adopted in standards like IEEE 802.16 WiMAX [6]. A significant reduction of the search space for the different interleaver parameters was achieved with an important improvement in TC error rate performance.

The rest of the paper is organized as follows. In Section II, a description of the considered interleaver model and interleaver design criteria is given. Section III introduces the proposed graph-based design method for TC interleavers. In Section IV, a puncturing pattern selection strategy is proposed, followed by the description of the constraints on the interleaver imposed by the puncturing patterns. Then, in Section $\mathrm{V}$, the proposed method is applied to design TC interleavers and puncturing patterns for a set of frame parameters included in LTE. Section VI shows the simulated error rate performance of the proposed code and its comparison with the original LTE code. Finally, Section VII concludes the paper.

\section{INTERLEAVER MODEL AND DESIGN CRITERIA}

In this study, TC interleaving is defined as follows: the interleaver reads the symbols from an input vector $\mathbf{d}$ and writes them to an output vector d' of size $K$ corresponding to the information frame length. A symbol read out from address $\Pi(i)$ in $\mathbf{d}$ is written to address $i$ in d', $\Pi$ denoting the interleaver function. When using Circular Recursive Systematic Convolutional (CRSC) codes [7] as constituent codes of the TC, $\mathbf{d}$ and d' can be represented by circles.

Three of the most popular interleaver families for TCs are the QPP interleavers [8] adopted in LTE [2], the DRP interleavers [9], and the ARP interleavers [5]. As shown in [10], the ARP is a sufficient permutation model to design TCs since it guarantees $d_{\min }$ values at least as high as the DRP and the QPP interleavers. Thus, our study only focuses on the ARP family but can also be generalized to the other families. The ARP interleaver structure is derived from the Regular Interleaver (RI):

$$
\Pi(i)=(P \cdot i) \bmod K
$$

where $P$ is the RI period that must be relatively prime to $K$. A degree of disorder is introduced into the permutation through a vector of shifts $\mathbf{S}$, leading to the ARP:

$$
\Pi(i)=(P \cdot i+S(i \bmod Q)) \bmod K .
$$

$\mathbf{S}$ has length $Q$ and to guarantee the bijectivity of the ARP interleaver function, $Q$ must be a divisor of $K$.

Two main criteria have to be considered for the design of a TC interleaver: the Hamming distance spectrum of the TC and the correlation between the channel information and extrinsic data at the input of each component decoder. Two measurable parameters related to these criteria have been considered: minimum span and correlation girth.

\section{A. Interleaver Design Criteria}

The increase of the minimum value of span $\left(S_{\min }\right)$ associated to the interleaver was shown to yield larger $d_{\text {min }}$ 
values for the resulting TC [11]. Thus, this value needs to be maximized. When using tail-biting termination, it was shown in [12] that the maximum achievable value for $S_{\min }$ has the following theoretical upper bound:

$$
S_{\min } \leq\lfloor\sqrt{2 K}\rfloor
$$

A correlation graph can be defined for the design of TC interleavers [13]. The correlation graph for TCs is a regular graph of degree $r=4$, since each data has two neighbors in d and d', with a number of vertices $x=K$. The interleaver should be then designed to maximize the correlation girth $g$ on this graph.

A theoretical upper bound on the girth value $g$ of a regular graph of degree $r$ is derived from the Moore bound [14]. Let $x(r, g)$ be the lowest number of vertices in a $r$-regular graph with girth $g . g$ can be at most proportional to the logarithm of $x(r, g)$ :

$$
g \leq 2 \log _{r-1}(x(r, g))+O(1)
$$

where $O(1)$ is the error term of the approximation. For the block size considered in this paper, $K=1504$, the theoretical upper bound on the correlation girth obtained from (4) is 13 .

\section{GRAPH-BASED CONSTRUCTION OF ARP}

In order to simplify the interleaver parameters selection, the interleaver structure is divided into different groups of addresses or layers that are progressively placed to complete the interleaver structure. From (2), it can be shown that:

$$
\Pi(i+Q) \bmod Q=\Pi(i) \bmod Q .
$$

Therefore, $Q$ groups of permutation addresses are identified in the whole interleaver size $K$, each one corresponding to a given modulo $Q$ value. Thus, the interleaver parameters can be selected by dividing the permutation addresses into $Q$ groups or layers with index $l=0, \ldots, Q-1$. In Fig. 1 the interleaver structure is divided into its $Q$ corresponding layers and represented in a circle with the addresses of $\mathbf{d}$ and $\mathbf{d}$ ' in its inner and outer parts, respectively. Note that the initial permutation addresses (i.e., non-shifted) are fixed by $P$.

The final permutation addresses of each layer $l$ are defined by choosing its corresponding shift value $S(l)$. The layer number $l$ to which address $i$ in d' belongs is determined as:

$$
l=i \bmod Q \text {. }
$$

For each layer $l$, we propose to express the shift value as:

$$
S(l)=T_{l}+A_{l} Q
$$

where $T_{l}=0, \ldots, Q-1$ and $A_{l}=0, \ldots,(K / Q)-1$ correspond to the inter and intra-layer shifts for layer $l$, respectively. The inter-layer shift $T_{l}$ defines the position that a given layer takes among the $Q$ different layers as shown in Fig. 2(a), whilst the intra-layer shift $A_{l}$ defines the rotation that a given layer performs inside the considered layer as shown in Fig. 2(b). Thus, the value of $S(l)$ can be obtained first by selecting the value of inter-layer shift $T_{l}$ and then selecting the value of intra-layer shift $A_{l}$. Note that the proposed shift selection

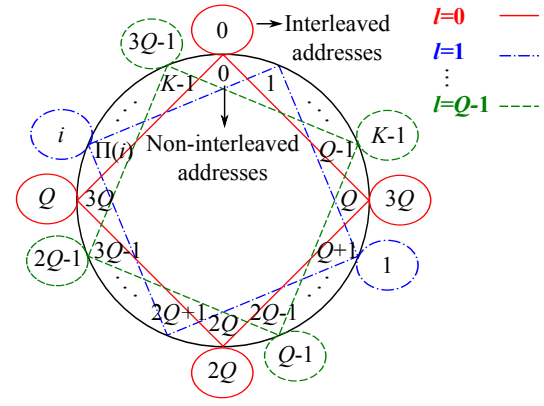

Fig. 1. Possible representation of the ARP interleaver structure, with $S(0), \ldots, S(Q-1)=0$ and $K=4 Q$, when using CRSC constituent codes.

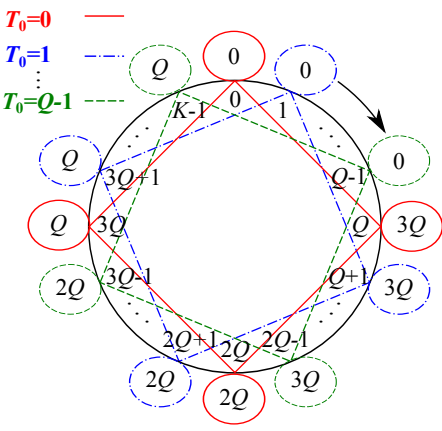

(a)

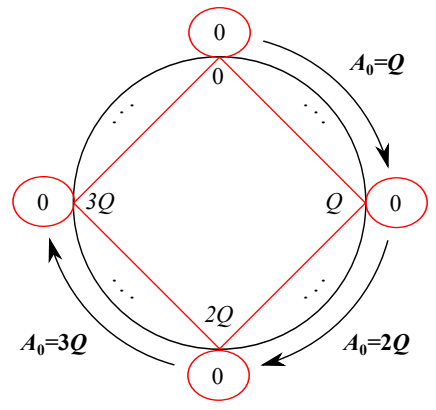

(b)
Fig. 2. (a) Different inter-layer shifts for layer $l=0$, with $K=4 Q$. (b) Possible intra-layer shifts for layer $l=0$, with $T_{0}=0$ and $K=4 Q$.

allows all possible positions for each layer to be considered. This graph-based construction of ARP interleavers simplifies the validation of minimum span and correlation girth criteria in the interleaver design, since these criteria are verified each time a new layer is placed.

Considering the above introduced shift selection method, for a given set of design parameters ( $S_{\min }$ and $g$ targets - chosen a few points below the upper bounds given by (3) and (4), $K$, $R$, polynomial generators, and puncturing mask), the proposed interleaver design strategy involves the following steps:

1) Select the candidate values for $\boldsymbol{P}$ : The set of admissible values for $P$ is the group of integers from 1 to $K-1$ relatively prime to $K$. Only the $C$ candidate values for $P$ ensuring a minimum span value greater than or equal to $S_{\text {min }}$ target, considering a RI structure (1), are selected.

2) Select the $\boldsymbol{Q}$ shift values for each candidate for $\boldsymbol{P}$ : For each candidate for $P$, layer $l$ is placed by computing a value for $S(l)$ from (7), fulfilling puncturing constraints if any (cf. Section IV). For this value, the $S_{\min }$ and $g$ are evaluated. If they are equal to or higher than $S_{\min }$ and $g$ targets, one can move on to layer $l+1$. Otherwise, another value for $S(l)$ has to be evaluated. This process is performed until the whole group of $Q$ shift values are determined.

3) Select the best ARP interleaver candidate: The best candidate for TC interleaver is selected from the group of candidates previously generated by comparing their Hamming distance spectra. The ARP interleaver candidate with the best TC Hamming distance spectrum is chosen. 


\section{Interleaving With PUnCturing Constraints}

As shown in [15], the puncturing of well-chosen systematic bits can increase $d_{\min }$ and reduce the convergence threshold of TCs. The selection of the systematic bits to be punctured must be performed carefully, in order to prevent the constituent codes of the TC from being affected by a catastrophic puncturing mask [3].

\section{A. Puncturing Mask Selection}

In this study, a periodic puncturing pattern with period $M$ is considered. The same CRSC code with coding rate $1 / 2$ is used for the two constituent codes of the TC. Thus, the puncturing mask is composed of two vectors of length $M$, corresponding to the puncturing positions in the data and parity vectors. The puncturing mask is defined according to the target code rate $R$ of the TC and to the puncturing period $M$. For given $R$ and $M$, the data puncturing rate $D P R$ can take $M+1$ different values: $D P R=\frac{m}{M}, m=0, \ldots, M$. However, the values of $D P R$ are restricted to those ensuring a CRSC code rate $R_{c}$ smaller than 1 , to be able to reconstruct $\mathbf{d}$ from the encoded sequence.

The puncturing mask design procedure proposed in this work involves the following steps:

1) Find the best puncturing pattern for each $D P R$ value: The best puncturing mask for each $D P R$ value is identified as the one generating the best CRSC Hamming distance spectrum (i.e., highest distance values in the first spectrum terms and minimal number of codewords at these distances).

2) Carry out a mutual information exchange analysis to select a restricted set of puncturing masks:

As pointed out in [15], conventional Extrinsic Information Transfer (EXIT) charts [16] are not appropriate to predict the convergence threshold of TCs with punctured data bits. Thus, an analysis of the real exchange of extrinsic information between the constituent Soft-In Soft-Out decoders, via uniform interleaving [17] within actual turbo decoding iterations, is carried out to select the best puncturing mask in terms of TC convergence. A similar analysis has previously been used to identify efficient precoding structures for TCs [18]. Unlike conventional EXIT charts, the crossing point of the curves in the modified EXIT charts is not the point of perfect convergence $(I A, I E)=(1,1)$. Therefore, the best puncturing masks in terms of convergence performance are selected as those providing a crossing point closer to $(1,1)$ than the mask with $D P R=0$.

Then, using the chosen puncturing masks, the error rate performance of the TC under uniform interleaving is analyzed. The mask providing the best tradeoff between convergence threshold and error floor performance is finally selected.

\section{B. Determination of Data and Parity Puncturing Constraints}

To avoid poor puncturing patterns in the second (interleaved) constituent CRSC code of the TC, a Data PunctureConstrained (DPC) interleaver must guarantee the same data puncturing pattern in both constituent CRSC codes [3].

During the turbo decoding process, extrinsic information from a given constituent decoder is generated based on its received parity sequence and is sent to the other constituent decoder via the interleaver/deinterleaver as a priori information on data. The extrinsic information computed from unpunctured parity positions is expected to be more reliable than the one generated from punctured parity positions. Therefore, a possible strategy for the interleaver construction involves connecting data positions associated with the most reliable extrinsic information from one constituent decoder to data positions associated with the least reliable extrinsic information in the other one. Under these conditions, the correction capability of the TC is spread over the data positions and the decoding of the less reliable positions should be improved.

For a given puncturing mask, the proposed connection strategy is defined by the following steps:

1) Identify free parities in a puncturing period $M$ :

Free parities are defined as unpunctured parities corresponding to unpunctured data symbols.

2) Identify error-prone data positions in a puncturing period $M$ : To this end, additional data puncturing is introduced at the remaining unpunctured data positions, and the resulting CRSC distance spectrum is evaluated. The number of additional punctured data positions corresponds to the number of free parities. The additional punctured data positions leading to the poorest CRSC distance spectrum (i.e., the lower $d_{\min }$ and the higher number of codewords at that distance or multiplicity) are then labeled as the most error-prone. Note that additional data puncturing is only introduced to identify error-prone data positions and is then removed from the puncturing mask.

3) Apply the connection strategy: The proposed strategy involves connecting free parities of a CRSC code to the most error-prone data positions of the other one.

In the ARP model, puncturing constraints are included via the $T_{l}$ values, to simplify their inclusion, $Q$ is set as a multiple of $M$ (in this study, $Q=M$ ). Since the layer order of the ARP interleaver is $Q$-periodic as shown by (5), the validation of puncturing constraints in a puncturing period $M$ is a sufficient condition for their validation in the whole data sequence.

In the following sections, an interleaver including the proposed parity puncturing constraint on top of the abovementioned data puncturing constraint is named Data and Parity Puncture-Constrained (DPPC) interleaver.

\section{Application Examples}

The previous design guidelines were applied to coding rates $2 / 3$ and $4 / 5$, for $K=1504$ and constituent codes CRSC(1, $15 / 13)_{8}$. Error rate performance was simulated in the AWGN channel with a BPSK modulation and a maximum of 16 decoding iterations with the maximum a posteriori probability (MAP) algorithm. Only the design for $R=2 / 3$ is detailed.

\section{A. Puncturing Mask Selection}

Puncturing mask design was carried out for puncturing period $M$ equal to 8 . Table I lists the distance spectrum of the CRSC constituent code for the best masks at each $D P R$ value. Only $D P R$ values with even values of $m$ were considered, leading to symmetric puncturing masks. The analysis of the 
distance spectra shows that $D P R$ values higher or equal to $6 / 8$ should be avoided. Indeed, the puncturing mask becomes catastrophic for the CRSC code.

Then, the convergence behavior with the different puncturing masks is analyzed in the TC structure. Fig. 3 shows the modified EXIT chart of the TC evaluated at the Signalto-Noise Ratio (SNR) decoding threshold of the code with puncturing mask $D P R=0$. The puncturing mask providing better convergence than the $D P R-0$ mask, due to its $(I A, I E)$ crossing point closer to $(1,1)$, corresponds to $D P R$ value $2 / 8$. In this example, since only one puncturing mask candidate remains, there is no need to plot the TC error rate performance under uniform interleaving to decide between them.

\section{B. Identification of Parity Puncturing Constraints}

In the selected puncturing mask, only the parity at position 1 is a free parity for $M=8$ (see Table I, data and parity positions range from 0 to 7 ). Table II lists the different distance spectra obtained including an additional punctured data symbol from positions 1 to 6 in the data puncturing mask 01111110. According to these results, 4 is the most error-prone data position in the puncturing period. Therefore, the DPPC interleaver must ensure that data symbols at positions 1 and 4 in $\mathbf{d}$ are interleaved to positions 4 and 1 in d', respectively.

\section{Puncture-Constrained Interleaver Design}

For $K=1504, S_{\min }$ has a theoretical upper bound of 54 as defined in (3). A $S_{\min }$ target of $80 \%$ of the $S_{\min }$ theoretical upper bound and a $g$ target of 8 were selected. Afterwards, the interleaver candidates are generated:

1) Selection of the candidate values for $\boldsymbol{P}$ : The maximum achievable value of $S_{\min }$ for $K=1504$ considering a RI structure is 52 . Thus, the best candidates for $P$ are those allowing a $S_{\min }$ value between 45 and 52 .

2) Selection of the $Q$ shift values for each candidate for $\boldsymbol{P}$ : The parameters for the ARP interleaver candidates are determined via the construction method presented in Section III. Three different design configurations have been studied. In the first one, the puncturing mask with Non Data-Punctured (NDP) $(D P R=0)$ is considered. In the other two, DPC, and DPPC ARP interleavers are considered for the $D P R-2 / 8$ mask. In order to compare the efficiency of the different configurations in finding large $d_{\text {min }}$ values, 64000 ARP candidates are generated by each configuration. The most efficient configuration is the one with the highest number of generated candidates meeting all the design constraints (girth, span and $d_{\text {min }}$ ).

3) Selection of the best ARP interleaver candidate: Table III lists the best ARP interleavers generated for each design configuration. For all candidates $S_{\min }=45$ and $g=8$. Their respective distance spectrum is estimated and given in Table IV. It is observed that the use of data puncturing allows to reach a larger $d_{\min }$ value. Furthermore, the proposed DPPC ARP interleaver allows a reduced $d_{\min }$ multiplicity compared to the DPC ARP interleaver.

Finally, Table $\mathrm{V}$ provides statistics on the search efficiency in finding parameter sets yielding large $d_{\min }$ values, for the
TABLE I

BEST CRSC DISTANCE SPECTRUM FOR EACH $D P R$, CORRESPONDING CODEWORD MULTIPLICITIES AT DISTANCE $d, \alpha(d)$, AND PUNCTURING MASKS $(0=$ PUNCTURED, $1=$ UNPUNCTURED $) . R=2 / 3, M=8$.

\begin{tabular}{c|c|c|c|c|c|c|c}
\hline$D P R$ & $R_{c}$ & $\alpha(0)$ & $\alpha(1)$ & $\alpha(2)$ & $\alpha(3)$ & $\alpha(4)$ & $\frac{\text { Data }}{\text { Parity }}$ \\
\hline \hline 0 & 0.8 & 0 & 0 & 0 & 15 & 89 & $\frac{11111111}{10100000}$ \\
\hline $\mathbf{2 / 8}$ & $\mathbf{0 . 8 8}$ & $\mathbf{0}$ & $\mathbf{0}$ & $\mathbf{3}$ & $\mathbf{6 2}$ & $\mathbf{5 6 6}$ & $\frac{\mathbf{0 1 1 1 1 1 0}}{\mathbf{1 1 0 0 0 0 0 1}}$ \\
\hline $4 / 8$ & 1 & 0 & 8 & 64 & 482 & 3616 & $\frac{11110000}{11100001}$ \\
\hline $6 / 8$ & 1.14 & 1 & 36 & 670 & 12122 & 219196 & $\frac{01000100}{11011100}$ \\
\hline
\end{tabular}

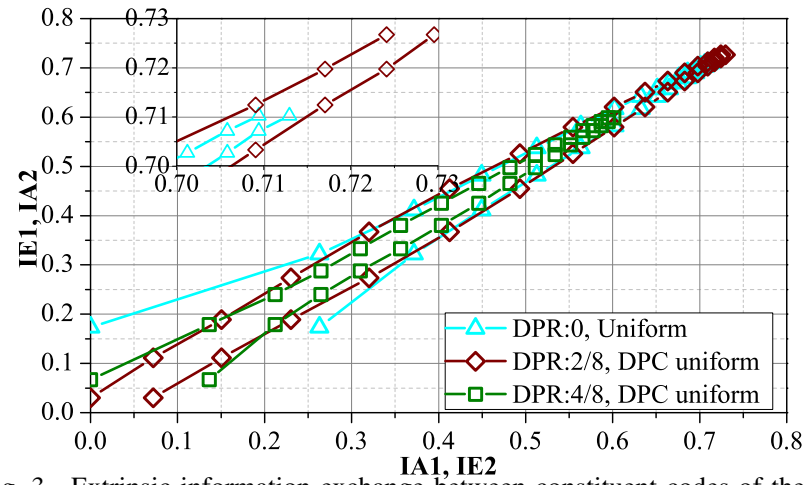

Fig. 3. Extrinsic information exchange between constituent codes of the TC at $\mathrm{E}_{\mathrm{b}} / \mathrm{N}_{0}=1.6 \mathrm{~dB}$ with $16 \mathrm{TC}$ iterations, $K=1504$, and $R=2 / 3$ under uniform or DPC uniform interleaving over the AWGN channel.

TABLE II

CRSC DISTANCE SPECTRUM OF THE $D P R$-2/8 MASK WHEN ONE ADDITIONAL DATA BIT IS PUNCTURED. $\alpha(d)$ IS THE MULTIPLICITY OF CODEWORDS AT DISTANCE $d$. CONSIDERED PARITY PUNCTURING MASK = $11000001(0=$ PUNCTURED, $1=$ UNPUNCTURED $)$.

\begin{tabular}{c|c|c|c|c|c}
\hline$\alpha(0)$ & $\alpha(1)$ & $\alpha(2)$ & $\alpha(3)$ & $\alpha(4)$ & Data Punc. Mask \\
\hline \hline 0 & 0 & 1880 & 1060320 & 465121494 & 00111110 \\
\hline 0 & 0 & 4000 & 2003510 & 671273377 & 01011110 \\
\hline 0 & 4 & 3015 & 1151175 & 294778989 & 01101110 \\
\hline $\mathbf{0}$ & $\mathbf{8}$ & $\mathbf{1 4 0}$ & $\mathbf{2 2 2 9}$ & $\mathbf{3 5 1 7 6}$ & 01110110 \\
\hline 0 & 2 & 2256 & 1275364 & 320347391 & 01111010 \\
\hline 0 & 4 & 3019 & 1152688 & 148963135 & 01111100 \\
\hline
\end{tabular}

TABLE III

BEST CANDIDATES FOR ARP INTERLEAVER WITH THE DIFFERENT CONFIGURATIONS, $S_{\min }=45, g=8, S(0)=0, R=2 / 3$ AND $K=1504$

\begin{tabular}{c|c|c|c|c|c|c|c|c}
\hline ARP & $P$ & $S(1)$ & $S(2)$ & $S(3)$ & $S(4)$ & $S(5)$ & $S(6)$ & $S(7)$ \\
\hline \hline NDP & 399 & 792 & 630 & 829 & 1010 & 90 & 1471 & 658 \\
\hline DPC & 227 & 495 & 998 & 280 & 1090 & 734 & 361 & 362 \\
\hline DPPC & 699 & 289 & 1452 & 1292 & 1349 & 391 & 417 & 874
\end{tabular}

TABLE IV

ESTIMATED DISTANCE SPECTRUM FOR THE BEST ARP INTERLEAVERS IN AWGN CHANNEL WITH CORRESPONDING MULTIPLICITIES $\alpha(d)$ AND CUMULATED INPUT WEIGHT AT $d_{\min }=d_{0}, w_{d_{0}} . R=2 / 3$ AND $K=1504$.

\begin{tabular}{c|c|c|c|c|c|c|c}
\hline ARP & $w_{d_{0}}$ & $d_{0}$ & $d_{1}$ & $d_{2}$ & $\alpha\left(d_{0}\right)$ & $\alpha\left(d_{1}\right)$ & $\alpha\left(d_{2}\right)$ \\
\hline \hline NDP & 5640 & 15 & 16 & 17 & 1128 & 4512 & 7708 \\
\hline DPC & 4324 & 19 & 20 & 21 & 752 & 1880 & 5264 \\
\hline DPPC & 2444 & 19 & 20 & 21 & 376 & 2444 & 3572 \\
\hline
\end{tabular}

NDP, DPC and DPPC configurations. The first line of the table provides the number of candidates meeting the $S_{\min }$ and $g$ targets that were obtained with each configuration. Note that the more the constraints included into the interleaver, the larger the number of candidates validating these criteria. Furthermore, the proposed DPPC interleaver structure increases the percentage of candidate interleavers with large $d_{\text {min }}$ values and reduces the average time to find such interleavers. The 
search becomes thus more efficient due to the different design constraints.

TABLE V

STATISTICS ON THE EFFICIENCY OF THE DIFFERENT ARP CONFIGURATIONS FOR $R=2 / 3, K=1504$, AND 64000 CANDIDATES, OBTAINED WITH CPU: INTEL CORE I5 $3.3 \mathrm{GHZ}$ AND RAM 8 Go.

\begin{tabular}{c|c|c|c}
\hline ARP & NDP & DPC & DPPC \\
\hline \hline Candidates found meeting $S_{\min }$ and $g$ targets & 3873 & 6803 & 9710 \\
\hline Percentage of candidates with $d_{\min } \geqslant 18(\%)$ & 0 & 1.95 & 3.09 \\
\hline $\begin{array}{c}\text { Average time to find a candidate } \\
\text { with } d_{\min } \geqslant 18(\min )\end{array}$ & - & 13.67 & 8.47 \\
\hline Number of candidates with $d_{\min }=19$ & 0 & 7 & 14 \\
\hline
\end{tabular}

\section{Simulated Performance Results}

Fig. 4 shows the FER performance in AWGN channel of the 8 -state $\operatorname{CRSC}(1,15 / 13)_{8}$ TC for $R=2 / 3, R=4 / 5$, and $K=1504$, with the three sets of parameters listed in Table IV. In addition, error rate simulation results for the original LTE [2] TC are included for comparison. We observe that DPC and DPPC interleavers achieve better asymptotic performance than the conventional NDP interleaver, while also displaying a convergence improvement. Compared to the puncturing pattern and the interleaver adopted in LTE, the proposed DPPC interleaver provides a gain of about 0.5 and $0.7 \mathrm{~dB}$ in convergence threshold for $R=2 / 3$ and $R=4 / 5$, respectively and almost 4 decades in error floor.

\section{CONCLUSION}

A new method to design Turbo Code (TC) interleavers is proposed, which calls for a joint optimization of puncturing patterns and interleaver function. Catastrophic puncturing masks for the constituent codes of the TC are early identified and ruled out in the selection process by evaluating their respective distance spectrum. Then, a modified EXIT chart analysis allows identifying suitable puncturing masks for the data punctured TC in terms of convergence performance.

When combined with a graph-based ARP model, it was shown that significant improvements in convergence threshold and error floor can be achieved by including data and parity puncturing constraints into the interleaver design. Furthermore, it was observed that adding stronger constraints made the interleaver parameter research process more efficient.

With the proposed method, turbo codes complying with the $5 \mathrm{G}$ requirements can now be easily designed.

\section{ACKNOWLEDGMENT}

This work has been performed in the framework of the Horizon 2020 project FANTASTIC-5G (ICT-671660) receiving funds from the European Union. The authors would like to acknowledge the contributions of their colleagues in the project, although the views expressed in this contribution are those of the authors and do not necessarily represent those of the project.

\section{REFERENCES}

[1] E. Dahlman, G. Mildh, S. Parkvall, J. Peisa, J. Sachs, and Y. Selen, "5G radio accsess," in Ericsson Review, June 2014. [Online]. Available: http://www.ericsson.com/res/thecompany/docs/publications/ ericsson_review/2014/er-5g-radio-access.pdf

[2] ETSI, "LTE Envolved Universal Terrestrial Radio Access(E-UTRA): Multiplexing and channel coding," TS 136212 (V10.0.0), January 2011.

[3] J.-F. Cheng, A. Nimbalker, Y. Blankenship, B. Classon, and T. Blankenship, "Analysis of circular buffer rate matching for LTE turbo code," in Proc. IEEE 68th Vehicular Technology Conference (VTC 2008-Fall), Sept. 2008, pp. 1-5.

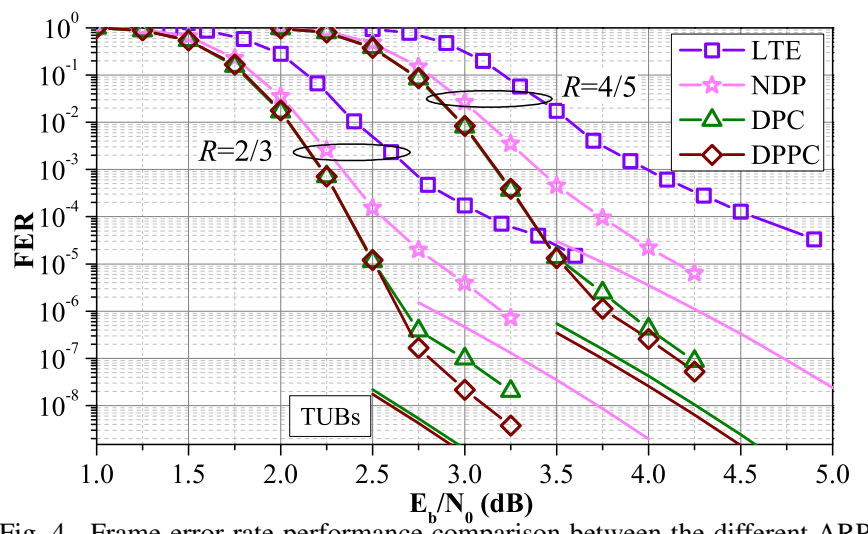

Fig. 4. Frame error rate performance comparison between the different ARP interleaver configurations and the LTE in AWGN channel with a max of 16 decoding iterations of the MAP algorithm for $R=2 / 3, R=4 / 5, K=1504$, and $\operatorname{CRSC}(1,15 / 13)_{8}$ constituent codes. TUB $=$ Truncated Union Bound.

[4] C. Berrou, A. Glavieux, and P. Thitimajshima, "Near Shannon limit error-correcting coding and decoding: Turbo-codes," in Proc. IEEE International Conference on Communications, (ICC'93), vol. 2, Geneva, Switzerland, May 1993, pp. 1064-1070.

[5] C. Berrou, Y. Saouter, C. Douillard, S. Kerouedan, and M. Jezequel, "Designing good permutations for turbo codes: towards a single model," in Proc. IEEE International Conference on Communications, (ICC'04), vol. 1, Paris, France, Jun. 2004, pp. 341-345.

[6] IEEE, "IEEE standard for local and metropolitan area networks, Part 16 Air interface for fixed and mobile broadband wireless access systems," IEEE Std 802.16-2004/Cor 1-2005, Feb. 2006.

[7] C. Weiss, C. Bettstetter, and S. Riedel, "Code construction and decoding of parallel concatenated tail-biting codes," IEEE Trans. Inf. Theory, vol. 47 , no. 1, pp. 366-386, Jan 2001.

[8] J. Sun and O. Takeshita, "Interleavers for turbo codes using permutation polynomials over integer rings," IEEE Trans. Inf. Theory, vol. 51, no. 1, pp. 101-119, Jan. 2005.

[9] S. Crozier and P. Guinand, "Distance upper bounds and true minimum distance results for turbo-codes designed with DRP interleavers," Annals of Telecommunications, vol. 60, no. 1-2, pp. 10-28, 2005.

[10] R. Garzón Bohórquez, C. Abdel Nour, and C. Douillard, "On the equivalence of interleavers for turbo codes," IEEE Wireless Commun. Lett., vol. 4, no. 1, pp. 58-61, Feb. 2015.

[11] S. Crozier, "New high-spread high-distance interleavers for turbo-codes," in Proc. 20th Biennial Symposium on Communications, Queen's University, Kingston, Ontario, Canada, May 2000, pp. 3-7.

[12] E. Boutillon and D. Gnaedig, "Maximum spread of D-dimensional multiple turbo codes," IEEE Trans. Commun., vol. 53, no. 8, pp. 12371242, Aug. 2005.

[13] Y. Saouter, "Selection procedure for binary turbocode permutation parameters," in Proc. 5th Int. Symposium on Turbo Codes and Related Topics, Laussane, Switzerland, Sept 2008, pp. 272-276.

[14] N. Biggs, "Minimal regular graphs with given girth," in Algebraic graph theory. New York, NY, USA: Cambridge University Press, 1974, pp. $180-190$.

[15] K. Gracie and S. Crozier, "Convergence performance and EXIT analysis of 4-state partially-systematic turbo codes," in Proc. 5th Int. Symposium on Turbo Codes and Related Topics, Sept. 2008, pp. 414-419.

[16] S. ten Brink, "Convergence behavior of iteratively decoded parallel concatenated codes," IEEE Trans. Commun., vol. 49, no. 10, pp. 1727 1737, Oct. 2001.

[17] S. Benedetto and G. Montorsi, "Unveiling turbo codes: some results on parallel concatenated coding schemes," IEEE Trans. Inf. Theory, vol. 42, no. 2, pp. 409-428, 1996

[18] R. Garzón Bohórquez, C. Abdel Nour, and C. Douillard, "Precoding techniques for turbo codes," in Proc. 21th European Wireless Conference: $5 G$ and Beyond (EW 2015), Budapest, Hungary, May 2015, pp. 1-6. 